\title{
On Minimum Sensing Error with Spectrum Sensing Using Counting Rule in Cognitive Radio Networks
}

\author{
Tao Jiang, Daiming Qu \\ Department of Electronics and Information Enginnering \\ Huazhong University of Science and Technology \\ Wuhan National Laboratory for Optoelectronics, Wuhan, 430074, China \\ Email: Tao.Jiang@ieee.org
}

\begin{abstract}
In cognitive radio networks (CRN), spectrum sensing requires each secondary user to efficiently and effectively detect the presence of primary users. In this paper, we study cooperative spectrum sensing (CSS), which is based on counting rules. Particularly, we first derive the optimal sensing strategy of CSS to minimize the sensing error, and then propose a simple algorithm to calculate the optimal parameter settings for CSS. Numerical results have been conducted and also validated that our proposed approach can achieve satisfying performance.
\end{abstract}

\section{Categories and Subject Descriptors}

C.2.1 [Network Architecture and Design]: Wireless communication

\section{General Terms}

Spectrum sensing, optimization, error

\section{Keywords}

Cognitive radio, cooperative spectrum sensing, counting rule, sensing error

\section{INTRODUCTION}

The demand of radio spectrum is increasing significantly in recent years. However, the majority of available spectrum resources has been allocated to licensed use, leaving limited room for new wireless services. It has been observed that, the average spectrum occupancy of the primary (licensed) spectrums is only $5.2 \%$ in current wireless communications systems [1]. This discrepancy motivates the idea of cognitive radio networks $[2,3]$, where secondary (unlicensed) users are

*Conference name: WICON'08, November 17-19, 2008, Maui, Hawaii, USA.

Copyright 2008 ICST 978-963-9799-36-3 allowed to transmit and receive data over unused primary (licensed) spectrum.

To avoid interference to primary users caused by secondary users, spectrum sensing, as a fundamental problem in cognitive radio networks, requires secondary users to efficiently and effectively detect the presence of the primary users. To limit the interference from secondary network to primary system, secondary network is intentionally designed to be far from primary transmitter, which results in low signal-tonoise-ratio (SNR) of the primary signals at the secondary detectors. In addition, fading and shadowing environments make spectrum sensing more involved. Longer sensing time can enhance sensing performance. However, secondary users can not transmit date over a primary channel if this channel is sensed. Otherwise, this primary channel will be sensed to be occupied by primary users even if it is not. Thus, longer sensing time will lead to shorter time of secondary data transmissions.

An alternative approach is to employ multiple secondary users to sense spectrum simultaneously. Cooperative spectrum sensing (CSS), where local sensors sense the existence of primary users and send information to the center, has been studied to improve sensing performance [4]-[8]. An AND rule based on CSS has been proposed in [4] and it also has been proven that CSS can significantly improve the sensing performance. Performance of CSS based on OR rule in a fading channel has been analyzed in [5]. In [6], link budget of CSS has been developed. To make the mathematical analysis more tractable, optimal methods based on a linear combination of the local sensing measurements are proposed in [7]. In [8], a liner-quadratic (LQ) strategy has been developed to combat the detriment effects of correlation between different secondary users.

As we know, AND rule and OR rule are two special cases of the counting rule, in which the network center makes decision according to the number of the secondary users that claiming the existence of primary users. The optimal linear cooperation for spectrum sensing proposed in [7] is simpler than counting rule, but it requires more data transmission between the secondary users and the network center. Although the performance of counting rule is worse than that of LQ detector when the correlation between the secondary users is strong, the former one announces a superior performance to the latter one in a weak-correlation case [8]. How- 
ever, according to the exponential correlation model, which has been proposed in [9], the correlation coefficient between two secondary users far apart about $60 \mathrm{~m}$ is only approximately 0.003 in an urban environment, and this weak correlation motivates a further study of counting rule. In this paper, we consider the counting rule as a fusion strategy of decisions of secondary detectors.

Regarding local sensing techniques, we consider both matched filter [10] and energy detection[11]. It is well known that matched filter is optimal when secondary users know the characteristics of primary signals, whereas the energy detection is a natural choice.

To the best of our knowledge, most of existing sensing objectives are based on Neyman-Pearson (NP) criterion [4]-[8]. One of the NP criterion is to maximize probability of detection $P_{d}$ with the constraint of maximum probability of false alarm $P_{f}$, where $P_{d}$ denotes the probability that a primary channel is sensed to be occupied when it is actually occupied, $P_{f}$ denotes the probability that a primary channel is sensed to be occupied when it is actually idle. Another of the NP criterion aims to minimize $P_{f}$ with the constraint of minimum $P_{d}$. According to these definitions, $1-P_{d}$ denotes the probability that sensing error happens when a channel is occupied and and $P_{f}$ denotes the probability that sensing error happens when a channel is idle. The total sensing error is a combination of $1-P_{d}$ and $P_{f}$. In this paper, we aim to minimize the sensing error under the constraint of the maximum probability of false alarm. Therefore, this sensing objective jointly consider the sensing error of $1-P_{d}$ or $P_{f}$.

In this paper, we deeply do research on the CSS using counting rule for cognitive radio networks. Firstly, we derive an optimal settings of sensing for both matched filter and energy detection to minimize the total sensing error. Then, we propose a simple algorithm to calculate the parameters of the optimal settings for CSS. Based on our theoretical analysis, the randomized rule and non-randomized rule, developed for distributed detection system, are also discussed in detail.

The rest of the paper is organized as follows. We first illuminate the system model in Section 2, and analyze the randomized and non-randomized counting rules in Section 3. We then develop the optimal strategy to minimize the total sensing error in Section 4, and simulation results are given and have been validated to our proposed schemes in Section 5. Finally, conclusions are drawn in Section 6.

\section{SYSTEM MODEL}

Consider $M$ sparsely dispersed secondary users in a secondary network whose size is smaller than the distance between the secondary network and the primary network. In this paper, we assume that: 1) the primary signals received by secondary users are identical and independent distributed (i.i.d.) random variables; 2) the noise power is constant for different secondary users, and 3) similar to $[4,5]$, the same decision rule is adopted for all secondary users.

Let $\mathcal{H}_{0}$ and $\mathcal{H}_{1}$ denote the absence and the presence of primary users, respectively. The main function of spectrum sensing is to make a decision between the following two hy- potheses:

$$
\begin{aligned}
& \mathcal{H}_{0}: y_{i}(k)=\varepsilon_{i}(k), \quad i=1, \ldots, M, \\
& \mathcal{H}_{1}: y_{i}(k)=h_{i} s(k)+\varepsilon_{i}(k), \quad i=1, \ldots, M,
\end{aligned}
$$

where $k=1, \ldots, N$, and $N$ is the sample size [11]. $y_{i}(k)$ is the signal received by the $i$ th secondary detector, and $s(k)$ is the signal transmitted by primary users. $h_{i}$ denotes the channel amplitude gain at the $i$ th sensor, and $\varepsilon_{i}$ is the zeromean additive white Gaussian noise noise (AWGN) at the $i$ th secondary user.

\subsection{Matched Filter}

It is well known that the optimal detector is matched filter in cognitive radio networks when the characteristics of primary signals, such as preambles, pilots and synchronization symbols, are obtainable for secondary detectors [12]. Let $P_{s}$ and $\sigma^{2}$ denote the signal power and noise power, respectively. $P_{d}^{m}$ and $P_{f}^{m}$ denote the probability of detection and probability of false alarm for matched filter, respectively. Thus, $P_{d}^{m}$ and $P_{f}^{m}$ can be formulated as [12]

$$
\begin{array}{r}
P_{f}^{m}=\mathcal{Q}\left(\lambda^{m}\right) \\
P_{d}^{m}=\mathcal{Q}\left(\lambda^{m}-\sqrt{\gamma}\right)
\end{array}
$$

where $\lambda^{m}$ is the threshold, and $\gamma=N \frac{P_{s}}{\sigma^{2}}$ denotes SNR. The definition of $Q(z)$ function is $Q(z)=\int_{z}^{\infty} \frac{1}{\sqrt{2 \pi}} \exp \left(-\frac{y^{2}}{2}\right) d y$.

In a fading channel, $P_{f}^{m}$ is the same as that in AWGN channel. The average probability of detection $P_{d}^{\prime m}$ may be derived by averaging (2) over fading statistics as

$$
P_{d}^{\prime m}=\int_{\gamma} \mathcal{Q}\left(\lambda^{m}-\sqrt{\gamma}\right) f(\gamma) d \gamma
$$

where $f(\gamma)$ is the probability density function (PDF) of SNR in fading channels.

\subsection{Energy detection}

When secondary users do not know the features of primary signals, the energy detection is a natural choice [13] as the optimal detector. The decision statistic for energy detection at the $i$ th secondary user can be formulated as $Y_{i}=$ $\frac{1}{2 N} \sum_{k=1}^{2 N}\left|y_{i}(k)\right|^{2}$, where $N$ is the time bandwidth product [13]. For simplicity, we omit the subscript "i" of $Y_{i}$ and PDF of $Y$ can be written as follows since $y_{i}$ is independent and identically distributed.

$$
\begin{array}{r}
f_{Y \mid \mathcal{H}_{0}}(y)=\frac{1}{2^{N} \Gamma(N)} y^{N-1} e^{-\frac{y}{2}} \\
f_{Y \mid \mathcal{H}_{1}}(y) 1=\frac{1}{2}\left(\frac{y}{2 \gamma}\right)^{\frac{N-1}{2}} e^{-\frac{2 \gamma+y}{2}} I_{N-1}(\sqrt{2 \gamma y})
\end{array}
$$

where $f_{Y \mid \mathcal{H}_{i}}(y)$ is the PDF of $y$ under $\mathcal{H}_{i}(i=0,1) . \Gamma(\cdot)$ is a gamma function and $I_{\nu}(\cdot)$ is the $\nu$ th order modified Bessel function of the first kind.

For energy detection, the probability of detection $P_{d}^{e}$ and 
the probability of false alarm $P_{f}^{e}$ can be evaluated as [13]

$$
\begin{array}{r}
P_{f}^{e}=P\left\{Y>\lambda^{e} \mid \mathcal{H}_{0}\right\}=\int_{\lambda}^{\infty} f_{Y \mid \mathcal{H}_{0}}(y) d y=\frac{\Gamma\left(N, \frac{\lambda}{2}\right)}{\Gamma(N)}(6) \\
P_{d}^{e}=P\left\{Y>\lambda^{e} \mid \mathcal{H}_{1}\right\}=\int_{\lambda}^{\infty} f_{Y \mid \mathcal{H}_{1}}(y) d y=Q_{N}(\sqrt{2 \gamma}, \sqrt{\lambda})(7)
\end{array}
$$

where $\lambda^{e}$ is the threshold, and $\Gamma(\cdot, \cdot)$ is the incomplete gamma function. $Q_{N}(\cdot, \cdot)$ is the generalized Marcum Q-function.

In a fading channel, the probability of detection is the same as that in AWGN channel. The detection probability in fading environments $P_{d}^{\prime}$ can be derived by averaging (7) as

$$
P_{d}^{\prime e}=\int_{\gamma} P_{d}^{e}(\gamma) f(\gamma) d \gamma
$$

The rest of this section is organized as follows. In Lemma 1 , we first give a proof the probability of detection is a concave function of probability of false alarm for matched filter and for energy detection when the channel is an AWGN. In Theorem 1, we prove these convexities also hold in fading environments.

LEMma 1. In an AWGN channel, given sampling size $N$ and SNR $\gamma, P_{d}^{m}$ is a concave function of $P_{f}^{m}$, and $P_{d}^{e}$ is a concave function of $P_{f}^{e}$.

PROOF: See Appendix A.

For simplify analysis, let $P_{d}$ denote the probability of detection and $P_{f}$ denote the probability of false alarm for either matched filter or energy detector in an AWGN channel. Similarly, $P_{d}^{\prime}$ denotes the probability of detection, and $P_{f}^{\prime}$ denotes the probability of false alarm for either matched filter or energy detector in a fading channel. Thus, we extend Lemma 1 to fading environments, as elaborated in Theorem 1.

Theorem 1. Given $N$, for any fading channels, $P_{d}^{\prime}$ is a concave function of $P_{f}^{\prime}$, and following inequalities hold.

$$
\begin{gathered}
\frac{d^{2} P_{d}^{\prime}}{d\left(P_{f}^{\prime}\right)^{2}}<0 . \\
\frac{1-P_{d}^{\prime}}{1-P_{f}^{\prime}}<\frac{d P_{d}^{\prime}}{d P_{f}^{\prime}}<\frac{P_{d}^{\prime}}{P_{f}^{\prime}} .
\end{gathered}
$$

PROOF: See Appendix B.

\section{ANALYSIS OF RANDOMIZED AND NON- RANDOMIZED COUNTING RULES}

For counting rule, we define $\Lambda$ as the number of secondary users that claim the existence of primary users. The decision strategy for counting rule can be expressed as

$$
\begin{cases}\text { if } \Lambda>K, & \text { decide } \mathrm{H}_{1}, \\ \text { if } \Lambda=K, & \text { decide } \mathrm{H}_{1} \text { with } \beta \text { probability, } \\ & \text { decide } \mathrm{H}_{0} \text { with } 1-\beta \text { probability, }(0 \leq \beta<1), \\ \text { if } \Lambda<K, & \text { decide } \mathrm{H}_{0},\end{cases}
$$

where $K=0,1, \ldots, M$ is the threshold at the center, and $\beta$ means if there are $K$ secondary users claim the existence of a primary user, the center decides the primary user exists with probability $\beta$. It is well known that when $\beta=0$, it is "non-randomized rule", and when $0<\beta<1$, we refer to it as "randomized rule" [16], [17].

In [16], it has been proven that the randomized tests can not be optimal in two sensor cases when local observations have continuous distributions; that is, the non-randomized rule can even be locally optimal if local (secondary user) observations have a continuous distributions. However, this proof has been found to be incorrect as explained in [17]. In [17], authors gave conditions for a randomized rule to be locally optimal and showed that a randomized fusion rule can be optimal with more restrictive assumptions than constraints in [16]. However, these more restrictive conditions described by several inequalities in [17] are too complicated to obtain explicit conclusions for a randomized fusion rule. In fact, it is of no significance for practical detection systems. Therefore, in this paper, we take a further step and derive some theoretical analysis results as follows.

According to the definitions of non-randomized and randomized rules, the final probability of false alarm $Q_{f}$ and final probability of detection $Q_{d}$ can be written as, respectively

$$
\begin{aligned}
Q_{f} & =\sum_{i=K+1}^{M} B\left(i ; M, P_{f}^{\prime}\right)+\beta B\left(K ; M, P_{f}^{\prime}\right), \\
Q_{d} & =\sum_{i=K+1}^{M} B\left(i ; M, P_{d}^{\prime}\right)+\beta B\left(K ; M, P_{d}^{\prime}\right),
\end{aligned}
$$

where $B(k ; n, p)=\left(\begin{array}{l}n \\ k\end{array}\right) p^{k}(1-p)^{n-k}$ and $B(M+1 ; M, x)=0$. For a given $N$ and $\gamma, P_{d}^{\prime}$ is a function of $P_{f}^{\prime}$. Obviously, both $Q_{f}$ and $Q_{d}$ are functions of $K, \beta$ and $P_{f}^{\prime}$ from (11) and (12). Therefore, in this paper, we aim to find the optimal $K, \beta$ and $P_{f}^{\prime}$ to minimize the sensing error.

THEOREM 2. The non-randomized rule is locally optimal if $P_{d}^{\prime}$ is a concave function of $P_{f}^{\prime}$.

PROOF: See Appendix C.

Theorem 2 means that the performance of "K-out-of-M" (non-randomized rules) points is better than that of their neighboring points (randomized rules). Furthermore, according to (34) and (35), for a given $K$ and $Q_{f}, Q_{d}$ is not a concave function of $\beta$ in the region of $\beta \in(0,1)$. That is to say, even if the randomized rule is optimal, it is still difficult to obtain the optimal $\beta$ of randomized rule solution by using iterative searching. Therefore, we consider the "K-out-of-M" (non-randomized) rules in the rest of this paper.

Fig. 1 and Fig. 2 illuminate $Q_{d}$ as a function of $K+1-\beta$ under an AWGN channel and a Rayleigh fading channel, respectively, where $Q_{f}=0.1, N=5, M=3$ and average $\gamma=$ 1.58. Under Rayleigh fading channel, the SNR $\gamma$ follows an exponential distribution given by [13, Eqn. (15)]

$$
f(\gamma)=\frac{1}{\bar{\gamma}} \exp \left(-\frac{\gamma}{\bar{\gamma}}\right)
$$




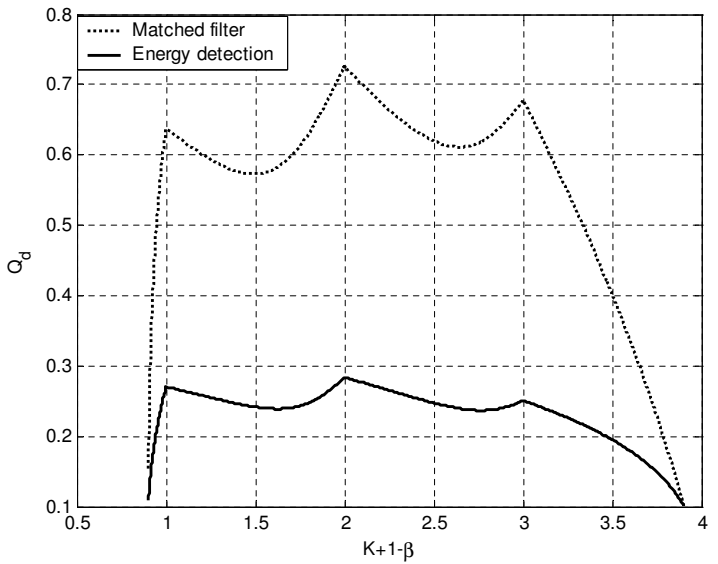

Figure 1: $Q_{d}$ as a function of $K+1-\beta$ under an AWGN with both matched filter and energy detection.

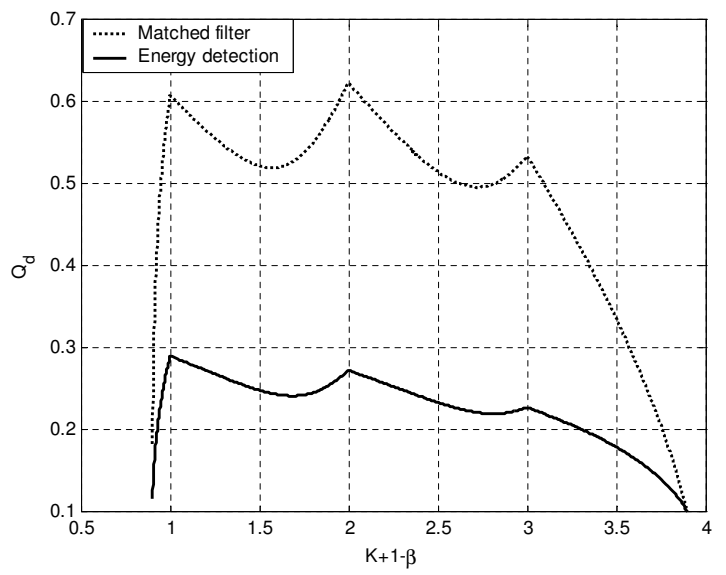

Figure 2: $\quad Q_{d}$ as a function of $K+1-\beta$ under a Rayleigh fading channel with matched filter and energy detection.

where $\bar{\gamma}$ is the average SNR. From Fig. 1 and Fig. 2, it is obvious that Theorem 2 has been validated. It also can be observed from Fig. 1 and Fig. 2 that matched filter has significantly better performance than that of energy detection.

The strategy of the "K-out-of-M" rules can be reformulated as follows

$$
\left\{\begin{array}{l}
\text { if } \Lambda>K, \quad \text { decide } \mathrm{H}_{1}, \\
\text { if } \Lambda \leq K, \quad \text { decide } \mathrm{H}_{0},
\end{array}\right.
$$

where $K=0,1, \ldots, M-1$. Then, $Q_{f}$ and $Q_{d}$ can be rewritten as

$$
Q_{f}=\sum_{i=K+1}^{M} B\left(i ; M, P_{f}^{\prime}\right)
$$

and

$$
Q_{d}=\sum_{i=K+1}^{M} B\left(i ; M, P_{d}^{\prime}\right)
$$

where $K=0,1, \ldots, M-1$. Therefore, we aim to find the optimal pair of $K$ and $P_{f}^{\prime}$ to minimize sensing error for cognitive radio networks.

\section{MINIMIZATION OF SENSING ERROR}

In this paper, we assume that $f(\gamma)$ is known to secondary users in cognitive radio networks. Obviously, this assumption is nonrestrictive since $f(\gamma)$ can be obtained in advance using long-time measurements. To derive the global optimal solution, we adopt a "divide and compare" strategy, we first obtain the local optimal $P_{f}^{\prime}$ to optimize the optimization objective of interest for every $K(0 \leq K \leq M-1)$, then compare them and choose the global optimal solution.

Let $c_{2}$ and $c_{1}=1-c_{2}$ denote the existence probability and absence probability of primary user, respectively. Therefore, the objective is to minimize the sensing error $\Phi=c_{1} Q_{f}+$ $c_{2}\left(1-Q_{d}\right)$. Thus, the sensing issue can be formulated as

$$
\begin{array}{rc}
\text { Minimize } & \Phi=c_{1} Q_{f}+c_{2}\left(1-Q_{d}\right), \\
\text { Subject to } & Q_{f} \leq \nu
\end{array}
$$

Since higher $Q_{f}$ leads to lower opportunity to potentially use idle channels, maximum probability of false alarm corresponds to minimum secondary system capacity. Let $\Upsilon_{\nu}$ denote the achieved secondary system capacity when $Q_{f}=\nu$, then the constraint $Q_{f} \leq \nu$ means the optimal solution of problem (15) should guarantee the corresponding secondary system capacity is not less than $\Upsilon_{\nu}$. We add this constraint based on the following principle: if $Q_{f}$ is too large, the secondary system capacity will be too small to make cognitive radio an attractive approach.

Considering

$$
\frac{d \Phi}{d P_{f}^{\prime}}=\pi_{1}\left(\pi_{2}-1\right)
$$

with

$$
\pi_{1}=-c_{1} M\left(\begin{array}{c}
M-1 \\
K
\end{array}\right) P_{f}^{\prime K}\left(1-P_{f}^{\prime}\right)^{M-K-1}<0
$$

and

$$
\pi_{2}=\frac{c_{2} P_{d}^{\prime K}\left(1-P_{d}^{\prime}\right)^{M-K-1}}{c_{1} P_{f}^{\prime K}\left(1-P_{f}^{\prime}\right)^{M-K-1}} \frac{d P_{d}^{\prime}}{d P_{f}^{\prime}}>0
$$

Therefore

Lemma 2. For $0 \leq K \leq M-1, \pi_{2}\left(P_{f}^{\prime}\right)$ is a monotonically decreasing function of $\overline{P_{f}^{\prime}}$.

PROOF: See Appendix D.

TheOrem 3. For a given $K$, when $\widetilde{P}_{f}$ is the root of $Q_{f}\left(P_{f}^{\prime}\right)=\nu$, $\widetilde{P}_{f}$ is the optimal solution of $(15)$ if $\pi_{2}\left(\widetilde{P}_{f}\right)>1$. If $\pi_{2}\left(\widetilde{P}_{f}\right)<1$, the root $\left(\bar{P}_{f}\right)$ of $\pi_{2}\left(P_{f}^{\prime}\right)=1$ is the optimal solution of $(15)$.

PROOF: See Appendix E. 
To obtain the optimal solution of (15), we need to calculate the root of equation $Q_{f}\left(P_{f}^{\prime}\right)=\nu$ or equation $\pi_{2}\left(P_{f}^{\prime}\right)=1$. Newton-Raphson method [18] can be employed to calculate the root of function $g(x)=0$ as follows:

1. Choose tolerance $\varepsilon$ and the initial guess $x(i)$, let $i=1$;

2. If $|g(x(i))|<\varepsilon$, stop; Otherwise, go to step 3;

3. Let $x(i+1)=x(i)-g(x(i)) / g^{\prime}(x(i))$, and $i=i+1$, go to step 2 .

Similarly, when a $K$ is given, the processing of calculating the optimal settings can be listed as follows:

1. Calculate the $\operatorname{root}\left(\widetilde{P}_{f}\right)$ of $Q_{f}\left(P_{f}^{\prime}\right)=\nu$. If $\pi_{2}\left(\widetilde{P}_{f}\right) \geq 1$, $\widetilde{P}_{f}$ is the optimal solution; else go to step 2;

2. Calculate the root $\left(\bar{P}_{f}\right)$ of $\Omega\left(P_{f}^{\prime}\right)=0$, and $\bar{P}_{f}$ is the optimal solution.

When we obtain the optimal solution for each $K$, the global optimization can be obtained by direct comparing them to choose the best one.

\section{SIMULATION RESULTS}

In this section, we present numerical results of total sensing error with different local probability of false alarm $P_{f}^{\prime}$. The simulation results are obtained using the following parameters: average SNR $\bar{\gamma}=8$, sample size $N=5$, number of local secondary detectors $M=4, c_{1}=0.4$ and $c_{2}=0.6$.

Fig. 3 and Fig. 4 demonstrate the total sensing error $\Phi$ varying with local probability of false alarm $P_{f}^{\prime}$ when matched filter is used under AWGN and Rayleigh-fading channel environments, respectively. Fig. 5 and Fig. 6 show the total sensing errors $\Phi$ are different when the local probability of false alarm $P_{f}^{\prime}$ is different when the energy detection is used under AWGN and Rayleigh-fading environments, respectively. From Fig $3 \sim$ Fig. 6 , it can be observed that for each $K=0,1,2,3$, there exists only minimum $\Phi_{\text {min }}$ for each case. In addition, $\Phi$ decreases with $P_{f}^{\prime}$ increasing when $\Phi<\Phi_{\min }$, and $\Phi$ increases with $P_{f}^{\prime}$ increasing when $\Phi>\Phi_{\min }$. Obviously, Theorem 2 can be validated. It also can be observed that the total sensing error, that is achieved by the optimal sensing settings, can be much lower than non-optimal sensing error.

Fig. 7 illuminates comparisons of $\Phi$ when matched filter and energy detection is used, respectively, in AWGN channel and Rayleigh-fading channel, respectively, for the threshold $K=1$. Similarly, Fig. 8 shows comparisons of $\Phi$ when matched filter and energy detection is used, respectively, in AWGN channel and Rayleigh-fading channel, respectively, for the threshold $K=2$. From Fig. 7 and Fig. 8, we can make some conclusions: 1) fading increases the sensing error, e.g., either matched filter or energy detection under Rayleigh fading channel have higher sensing error; 2) the matched filter has lower sensing error than that of energy detection under the same fading channel.

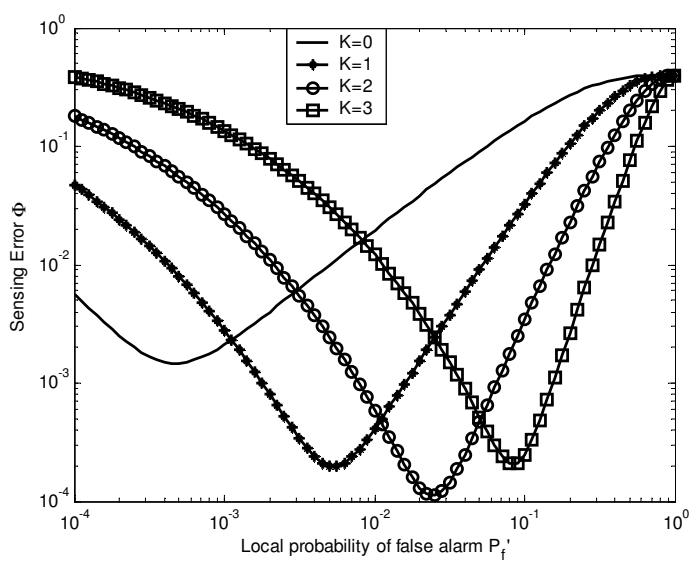

Figure 3: Sensing error $\Phi$ as function of local probability of false alarm $P_{f}^{\prime}$ under an AWGN channel with matched filter.

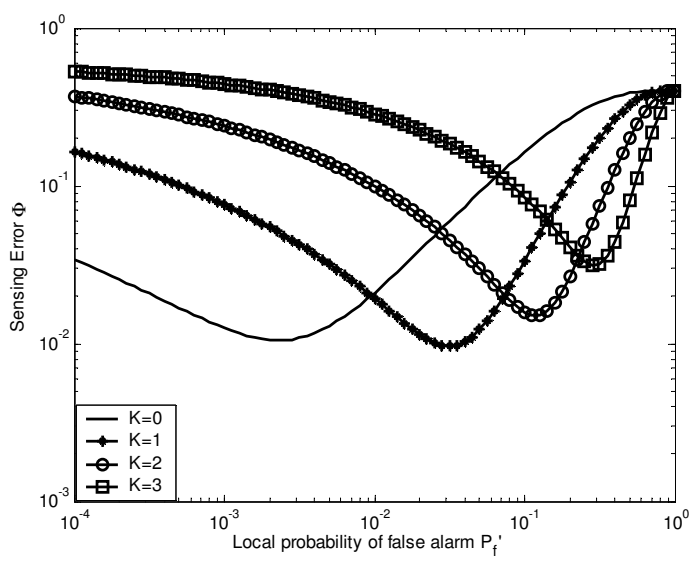

Figure 4: Sensing error $\Phi$ as function of local probability of false alarm $P_{f}^{\prime}$ under a Rayleigh fading channel with matched filter.

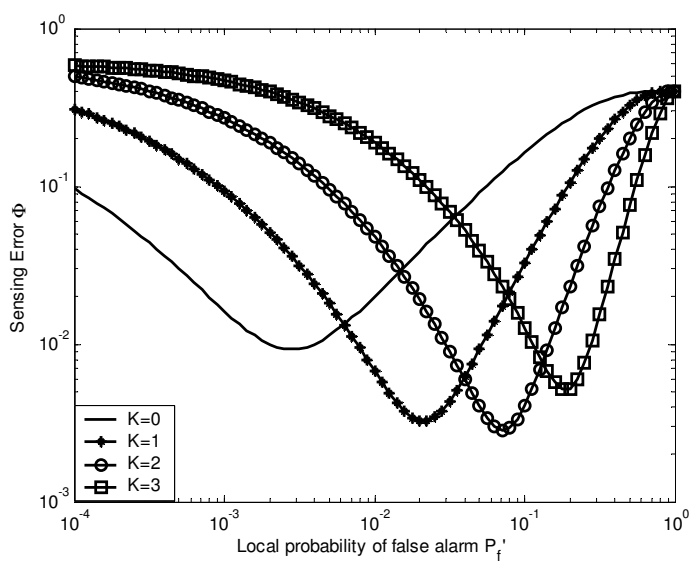

Figure 5: Sensing error $\Phi$ as function of local probability of false alarm $P_{f}^{\prime}$ under an AWGN channel with energy detection. 


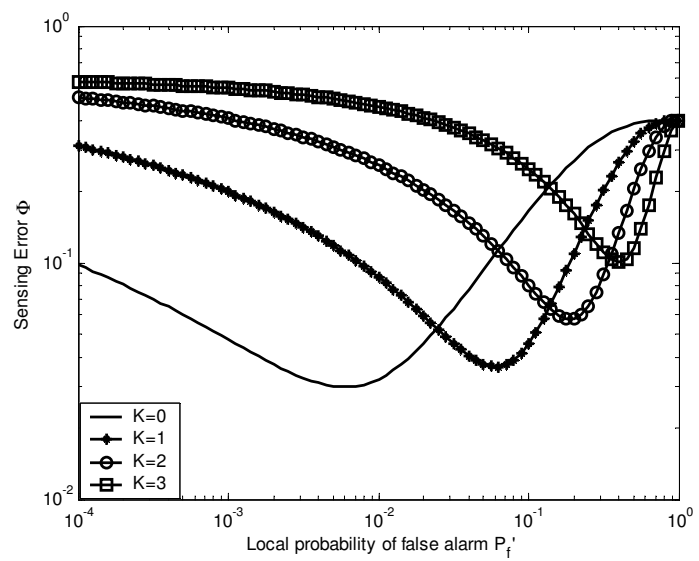

Figure 6: Sensing error $\Phi$ as function of local probability of false alarm $P_{f}^{\prime}$ under a Rayleigh fading channel with energy detection.

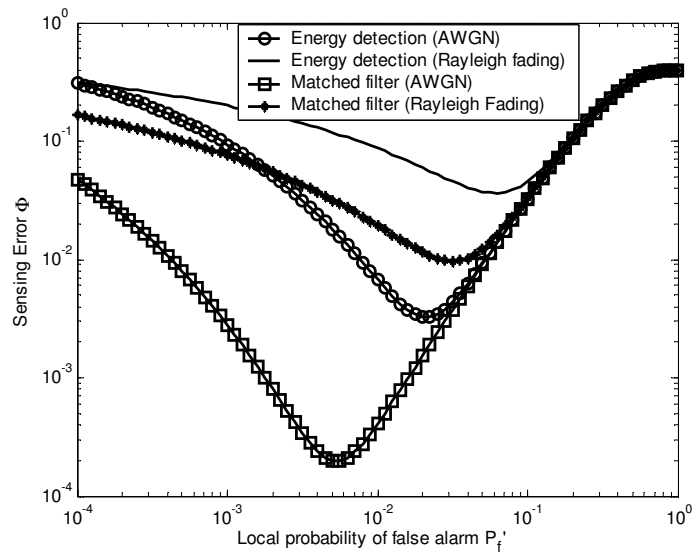

Figure 7: Comparisons of matched filter and energy detection under AWGN and Rayleigh-fading channels with threshold $K=1$.

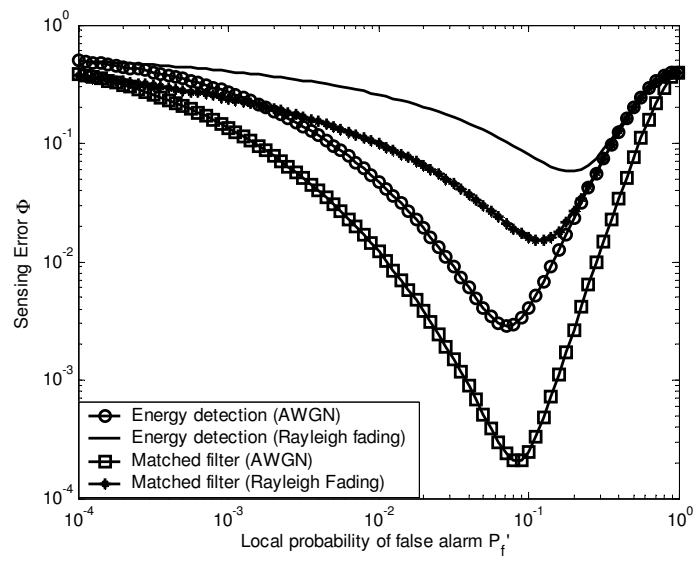

Figure 8: Comparisons of matched filter and energy detection under AWGN and Rayleigh-fading channels with threshold $K=2$.

\section{CONCLUSION}

In this paper, we investigated the optimal CSS strategy that employs counting rule to minimize the total sensing error for cognitive radio networks. The main contributions of this paper include: 1) it has been proved the probability of detection is a convex function for either matched filter or energy detection; 2) we analyzed the randomization at the center and found that the non-randomized rules are locally optimal for the i.i.d. signals in cognitive radio networks; 3 ) we derived the optimal counting rule for both matched filter and energy detection, respectively; 4) An easy-implemented algorithm has been developed to calculate the optimal settings of sensing error in cognitive radio networks. Simulation results show that the optimal sensing settings considerably outperforms the non-optimal settings in terms of sensing error.

\section{ACKNOWLEDGMENTS}

This work is partially supported by the NSF of China with Grants 60872008 and 60702039, the Program for New Century Excellent Talents in University of China with Grant NCET-08-0217, and the Research Fund for the Doctoral Program of Higher Education of the Ministry of Education of China under Grant 200804871142.

\section{REFERENCES}

[1] M. A. McHenry. NSF Spectrum Occupancy Measurements Project Summary. Shared Spectrum Company Report.

[2] J. Mitola and G. Q. Maguire. Cognitive radios: making software radios more personal. IEEE Personal Communications, 6(4): 13-18, Aug. 1999.

[3] S. Haykin. Cognitive radio: brain-empowered wireless communications. IEEE Trans. Commun., 23(2): 201-220, Feb. 2005.

[4] E. Visotsky, S. Kuffner, and R. Peterson. On collaborative detection of $\mathrm{tv}$ transmissions in support of dynamic spectrum sensing. In Proc. First IEEE Symposium on Dynamic Spectrum Access Networks, (Baltimore, USA, Nov. 2005), pages 131-136.

[5] A. Ghasemi and E. S. Sousa. Collaborative spectrum sensing for opportunistic access in fading environments,. In Proc. First IEEE Symposium on Dynamic Spectrum Access Networks, (Baltimore, USA, Nov. 2005), pages 338-345.

[6] S. M. Mishra, A. Sahai, and R. W. Broderson. Cooperative sensing among cognitive radios. In Proc. IEEE Int. Conf. Communications, (Istanbul, Turkey, June. 2006), pages 1658-1663.

[7] Z. Quan, S. Cui, and A. H. Sayed. Optimal linear cooperation for spectrum sensing in cognitive radio networks. IEEE J. Select. Signal Processing., 2(1): 28-40, Feb. 2008.

[8] J. Unnikrishnan and V. V. Veeravalli. Cooperative sensing for primary detection in cognitive radio. IEEE J. Select. Signal Processing., 2(1): 18-27, Feb. 2008.

[9] M. Gudmundson. A correlation model for shadow fading in mobile radio. Electr. Letters, 27(23):2145-2146, Nov. 1991.

[10] A. Sahai, N. Hoven, and R. Tandra. Some fundamental limits on cognitive radio. In Allerton Conference on Communication, Control, and 
Computing, (Oct. 2004.), pages 1658-1663.

[11] H. Urkowitz. Energy detection of unkown deterministic signals. Proceedings of the IEEE, 55(4): 523-531, April 1967.

[12] S. M. Kay. Fundamentals of Statistical Signal Processing, Detection Theory. Prentice-Hall, Upper Saddle River, NJ, 1993.

[13] F. F. Digham, M.-S. Alouini, and M. K. Simon. On the energy detection of unknown signals over fading channels. In Proc. IEEE Int. Conf. Communications (May 2003), pages 3575-3579.

[14] I. S. Gradshtein and I. Ryzhik. Table of Integrals, Series, and Products. Academic, San Diego, CA, 2000.

[15] S. Boyd and L. Vandenberghe. Convex Optimization. Cambridge Univ. Press.

[16] P. Willett and D. Warren. The suboptimality of randomized tests in distributed and quantized detection systems. IEEE Trans. Inform. Theory, 38(2): 355-361, Mar. 1992

[17] Y. I. Han and T. Kim. Randomized fusion rules can be optimal in distributed neyman-pearson detectors. IEEE Trans. Inform. Theory, 43(4): 1281-1288, July 1997.

[18] D. V. Griffiths and I. M. Smith. Numerical Methods for Engineers. Blackwell Scientific, London, 2002.

\section{APPENDIX}

\section{A. PROOF OF LEMMA 1}

First, we consider the matched filter. We get the proof that secondary derivative of $P_{d}^{m}$ over $P_{f}^{m}$ is negative, which is equivalent to prove $P_{d}^{m}$ is a concave function of $P_{f}^{m}$.

According to (1) and (2), we have

$$
\begin{array}{r}
\frac{d P_{f}^{m}}{d \lambda^{m}}=-\frac{1}{2 \pi} \exp \left(-\frac{\left(\lambda^{m}\right)^{2}}{2}\right) \\
\frac{d P_{d}^{m}}{d \lambda^{m}}=-\frac{1}{2 \pi} \exp \left(-\frac{1}{2}\left(\lambda^{m}-\sqrt{\gamma}\right)^{2}\right)
\end{array}
$$

Define $\rho^{m}=d P_{d}^{m} / d P_{f}^{m}$, combining (17) and we have (18), we have

$$
\rho^{m}=\frac{d P_{d}^{m} / d \lambda^{m}}{d P_{f}^{m} / d \lambda^{m}}=\exp \left(\sqrt{\gamma} \lambda^{m}-\frac{\gamma}{2}\right)
$$

Therefore

$$
\frac{d \rho^{m}}{d \lambda^{m}}=\sqrt{\gamma} \exp \left(\sqrt{\gamma} \lambda^{m}-\frac{\gamma}{2}\right)
$$

Then, the secondary derivative of $P_{d}^{m}$ over $P_{f}^{m}$ can be formulated as

$$
\frac{d^{2}\left(P_{d}^{m}\right)}{d\left(P_{f}^{m}\right)^{2}}=\frac{d \rho^{m} / \lambda^{m}}{d P_{f}^{m} / d \lambda^{m}}
$$

Note that, (18) implies $d P_{f}^{m} / d \lambda^{m}<0$ and (20) means $d \rho^{m} / \lambda^{m}>$ 0 . Therefore, $d^{2}\left(P_{d}^{m}\right) / d\left(P_{f}^{m}\right)^{2}<0$, and $P_{d}^{m}$ is a concave function of $P_{f}^{m}$.

Second, we consider the energy detection. Similarly, we prove secondary derivative of $P_{d}^{e}$ over $P_{f}^{e}$ is negative. Define $\rho^{e}=d P_{d}^{e} / d P_{f}^{e}$, according to (6) and (7), we have

$$
\rho^{e}=\frac{d P_{d}^{e} / d \lambda^{e}}{d P_{f}^{e} / d \lambda^{e}}=\frac{f_{Y \mid \mathcal{H}_{1}}\left(\lambda^{e}\right)}{f_{Y \mid \mathcal{H}_{0}}\left(\lambda^{e}\right)} .
$$

Note that [14, Section 8.44]

$$
I_{N-1}\left(\sqrt{2 \gamma \lambda^{e}}\right)=\left(\frac{\gamma \lambda^{e}}{2}\right)^{\frac{N-1}{2}} \sum_{k=0}^{\infty} \frac{\left(\frac{\gamma \lambda^{e}}{2}\right)^{k}}{k ! \Gamma(N+k)},
$$

Substituting (23), (6) and (7) into (22), we have

$$
\rho^{e}=e^{-\gamma} \Gamma(N) \sum_{k=0}^{\infty} \frac{\left(\frac{\gamma \lambda^{e}}{2}\right)^{k}}{k ! \Gamma(N+k)} .
$$

Obviously, $d \rho^{e} / d \lambda^{e}>0$, and then $\frac{d P_{f}^{e}}{d \lambda^{e}}=-f_{Y \mid \mathcal{H}_{0}}\left(\lambda^{e}\right)<0$.

Therefore, we can prove that

$$
\frac{d^{2} P_{d}^{e}}{d\left(P_{f}^{e}\right)^{2}}=\frac{d \rho^{e}}{d P_{f}^{e}}=\frac{d \rho^{e} / d \lambda^{e}}{d P_{f}^{e} / d \lambda^{e}}<0
$$

Therefore, $P_{d}^{e}$ is a concave function of $P_{f}^{e}[15]$.

\section{B. PROOF OF THEOREM 1}

Proof of (9) is equivalent to the proof that $P_{d}^{\prime}$ is a concave function of $P_{f}^{\prime}$.

First, we prove (9) as follow.

Combined (1) with (2) or combined (6) with (7), it is easy to find that $P_{d}$ is a function of $P_{f}$ and $\gamma$. Since integral can be represented as an infinite sum of integrand, we can write $P_{d}^{\prime}$ as

$$
P_{d}^{\prime}\left(P_{f}\right)=\int_{\gamma} P_{d}\left(\gamma, P_{f}\right) f(\gamma) d \gamma=\sum_{i=1}^{\infty} P_{d}\left(\gamma_{i}, P_{f}\right) f\left(\gamma_{i}\right),
$$

where $0<\gamma_{1}<\gamma_{2}<\ldots<\infty, 0<f\left(\gamma_{i}\right)<1$, and $\sum_{i=1}^{+\infty} f\left(\gamma_{i}\right)=1$ with $i=1,2, \ldots, \infty$.

Therefore, we have

$$
\frac{d^{2} P_{d}^{\prime}}{d P_{f}^{\prime 2}}=\frac{d^{2} P_{d}^{\prime}}{d P_{f}^{2}}=\sum_{i=1}^{+\infty} \frac{d^{2} P_{d}\left(\gamma_{i}, P_{f}\right)}{d P_{f}^{2}} f\left(\gamma_{i}\right) .
$$

Recalling Lemma 1, we have $\frac{d^{2} P_{d}\left(\gamma, P_{f}\right)}{d P_{f}^{2}}<0$ for any $\gamma$, we have

$$
\frac{d^{2} P_{d}^{\prime}}{d P_{f}^{\prime 2}}<0
$$

In above proven, we do no specify the distribution of fading channels, thus the conclusion is valid for any fading channels. Therefore, (9) has been proven and $P_{d}^{\prime}$ is a concave function of $P_{f}^{\prime}$.

(9) means $P_{d}^{\prime}$ is a concave function of $P_{f}^{\prime}$. After the proof of (9), (10) can be easily illustrated from Fig. 9. 


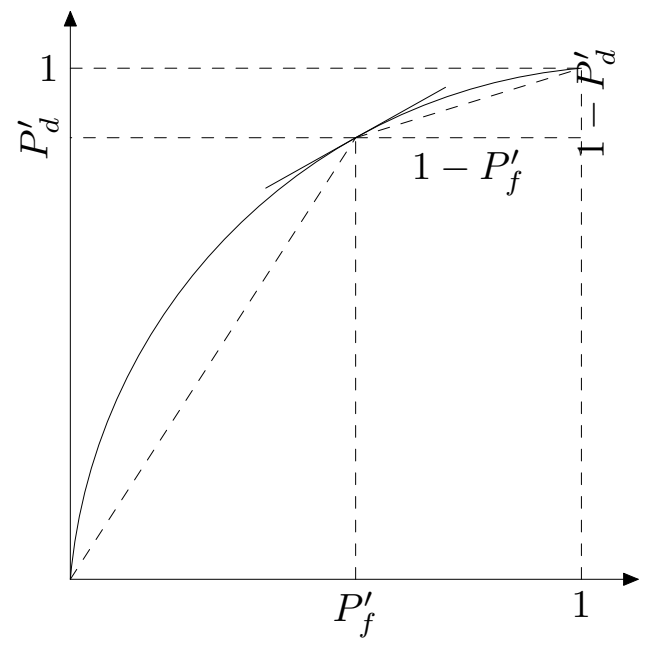

Figure 9: Geometrical explanation of (10)

\section{PROOF OF THEOREM 2}

Given $K$ and fixed $Q_{f}, P_{f}^{\prime}$ can be expressed as a function of $\beta$ (11). Note that, $P_{d}^{\prime}$ is a function of $P_{f}^{\prime}$ and $Q_{d}$ is a function of $P_{d}^{\prime}$. Therefore, $Q_{d}$ is a function of $\beta$. Let $\Gamma=\frac{d Q_{d}}{d \beta}$, and proving Theorem 2 is equivalent to proving the following two inequalities for a given $0 \leq K \leq M$ and $Q_{f}$ :

$$
\begin{gathered}
\Gamma_{\beta \rightarrow 0}<0, \\
\Gamma_{\beta \rightarrow 1}>0 .
\end{gathered}
$$

For

$$
U(x)=\sum_{i=k+1}^{M}\left(\begin{array}{c}
M \\
i
\end{array}\right) x^{i}(1-x)^{M-i}
$$

With

$$
\left(\begin{array}{c}
M \\
K
\end{array}\right)(M-K)=\left(\begin{array}{c}
M \\
K+1
\end{array}\right)(K+1)
$$

Then, we have

$$
U^{\prime}(x)=M\left(\begin{array}{c}
M-1 \\
K
\end{array}\right) x^{K}(1-x)^{M-K-1}
$$

Observing the first item on the right side of (11) and we can find that is is in the form of $U(x)$. Taking the first derivative of both sides of (11) with respect to $\beta$, we have

$$
\frac{d P_{f}^{\prime}}{d \beta}=-\frac{1}{(1-\beta)(M-K) \frac{1}{1-P_{f}^{\prime}}+\beta \cdot K \frac{1}{P_{f}^{\prime}}}
$$

Taking the first derivative of $Q_{d}$ with respect to $\beta$ in (12), we have

$$
\Gamma=\delta\left(\left((1-\beta)(M-K) \frac{1}{1-P_{d}^{\prime}}+\beta K \frac{1}{P_{d}^{\prime}}\right) \frac{d P_{d}^{\prime}}{d P_{f}^{\prime}} \frac{d P_{f}^{\prime}}{\beta}-1\right),
$$

with

$$
\delta=\left(\begin{array}{c}
M \\
K
\end{array}\right) P_{d}^{\prime K}\left(1-P_{d}^{\prime}\right)^{M-K}>0
$$

Substituting (31) into (32), we have

$$
\Gamma=\delta^{\prime}\left(\frac{(1-\beta)(M-K)}{1-P_{d}^{\prime}} b_{1}+\frac{\beta K}{P_{d}^{\prime}} b_{2}\right),
$$

with

$$
\begin{gathered}
\delta^{\prime}=-\delta \frac{1}{(1-\beta)(M-K) \frac{1}{1-P_{f}^{\prime}}+\beta K \frac{1}{P_{f}^{\prime}}}<0 \\
b_{1}=\frac{d P_{d}^{\prime}}{d P_{f}^{\prime}}-\frac{1-P_{d}^{\prime}}{1-P_{f}^{\prime}}
\end{gathered}
$$

and

$$
b_{2}=\frac{d P_{d}^{\prime}}{d P_{f}^{\prime}}-\frac{P_{d}^{\prime}}{P_{f}^{\prime}}
$$

According to (10), we know that $b_{1}>0$ and $b_{2}<0$.

Therefore, we have

$$
\begin{array}{r}
\Gamma_{\beta \rightarrow 0}=\delta^{\prime} \frac{M-K}{1-P_{d}^{\prime}} b_{1}<0 \\
\Gamma_{\beta \rightarrow 1}=\delta^{\prime} \frac{K}{P_{d}^{\prime}} b_{2}>0
\end{array}
$$

Therefore, Theorem 2 has been proven.

\section{PROOF OF LEMMA 4}

According to above analysis, the first derivative of $\pi_{2}\left(P_{f}^{\prime}\right)$ with respect to $P_{f}^{\prime}$ can be evaluated by

$$
\frac{d \pi_{2}}{d P_{f}^{\prime}}=\pi_{2} \times \omega
$$

with

$$
\begin{aligned}
\omega= & \frac{d^{2} P_{d}^{\prime}}{d P_{f}^{\prime 2}} \frac{d P_{f}^{\prime}}{d P_{d}^{\prime}}+K\left(\frac{1}{P_{d}} \frac{d P_{d}^{\prime}}{d P_{f}^{\prime}}-\frac{1}{P_{f}^{\prime}}\right) \\
& -(M-K-1)\left(\frac{1}{1-P_{d}^{\prime}} \frac{d P_{d}^{\prime}}{d P_{f}^{\prime}}-\frac{1}{1-P_{f}^{\prime}}\right) .
\end{aligned}
$$

According to (9), the first item in (37) is negative. Moreover, according to (10), we can also prove that the second and third items of (37) are negative. Therefore, $d \pi_{2} / d P_{f}^{\prime}<0$ is a monotonically decreasing function of $P_{f}^{\prime}$.

\section{E. PROOF OF THEOREM 3}

Consider

$$
\begin{aligned}
\lim _{P_{f}^{\prime} \rightarrow 0} \pi_{2}\left(P_{f}^{\prime}\right) & =\frac{c_{1}}{c_{2}}\left(\lim _{P_{f}^{\prime} \rightarrow 0} \frac{P_{d}^{\prime}}{P_{f}^{\prime}}\right)^{K} \lim _{P_{f}^{\prime} \rightarrow 0} \frac{d P_{d}^{\prime}}{d P_{f}^{\prime}} \\
& =\frac{c_{1}}{c_{2}}\left(\lim _{P_{f}^{\prime} \rightarrow 0} \frac{d P_{d}^{\prime}}{d P_{f}^{\prime}}\right)^{K+1} .
\end{aligned}
$$


According to (26), we have

$$
\lim _{P_{f}^{\prime} \rightarrow 0} \frac{d P_{d}^{\prime}}{d P_{f}^{\prime}}=\lim _{P_{f}^{\prime} \rightarrow 0} \int_{\gamma} \frac{d P_{d}}{d P_{f}} f(\gamma) d \gamma=\int_{\gamma} \lim _{P_{f}^{\prime} \rightarrow 0} \frac{d P_{d}}{d P_{f}} f(\gamma) d \gamma .
$$

According to (24), we also have $\lim _{P_{f}^{\prime} \rightarrow 0} \frac{d P_{d}}{d P_{f}}=\lim _{\bar{\lambda} \rightarrow+\infty} \rho=$ $+\infty$.

Since this inequality holds for any $\gamma$, from (39), we have $\lim _{P_{f}^{\prime} \rightarrow 0} \frac{d P_{d}^{\prime}}{d P_{f}^{\prime}} \rightarrow+\infty$. Combining this result with (38), we have $\lim _{P_{f}^{\prime} \rightarrow 0} \pi_{2}\left(P_{f}^{\prime}\right) \rightarrow+\infty$.

Let $\Omega\left(P_{f}^{\prime}\right)=\pi_{2}-1$, we can prove that $\lim _{P_{f}^{\prime} \rightarrow 0} \Omega\left(P_{f}^{\prime}\right)>0$.

If $\pi_{2}\left(\widetilde{P}_{f}\right)>1$, according to Lemma 2 , we have $\Omega\left(P_{f}^{\prime}\right)>0$ in range $P_{f}^{\prime} \in\left(0, \widetilde{P}_{f}\right)$. As a result, $\Phi$ is a decreasing function in the range $P_{f}^{\prime} \in\left(0, \widetilde{P}_{f}\right)$ according to (16). Therefore, we prove that $\widetilde{P}_{f}$ is the solution of (15).

If $\pi_{2}\left(\widetilde{P}_{f}\right)<1, \Phi$ is a decreasing function in $P_{f}^{\prime} \in\left(0, \bar{P}_{f}\right)$ but an increasing function in $P_{f}^{\prime} \in\left(\bar{P}_{f}, \widetilde{P}_{f}\right)$. Therefore, $\bar{P}_{f}$ is the solution of (15). 